\title{
THE DECONVOLUTION METHOD OF FAST PULSE SHAPING AT HADRON COLLIDERS
}

S. Gadomski1 ${ }^{1}$ G. Hall 2 , T. Høgh ${ }^{3}$, P. Jalocha ${ }^{1}$, E. Nygård ${ }^{4,5}$ and P. Weilhammer 5

\begin{abstract}
We describe a new technique for front end signal processing of signals from LHC or SSC detectors which precisely defines the origin of an event in time while maintaining amplitude measurement with an excellent signal to noise ratio.

The method is designed for use with silicon detectors whose leakage currents may be substantially increased during the lifetime of an experiment by radiation damage, although it is likely to be applicable to other types of detector. It makes use of a shaping amplifier with a time constant of several beam crossing intervals and is particularly well matched to CMOS front ends where low power consumption and low noise is best achieved by utilising pulse shapes with time constants $\sim 50 \mathrm{nsec}$.

It is based on discrete time filtering of data extracted from an analogue pipeline after a first level trigger. A finite impulse response type filter deconvolutes the sampled voltages of a shaped pulse to retrieve the original impulse signal with high precision. We describe the mathematical basis of the technique and its implications for timing and signal to noise.

Measurements have been made on a CMOS amplifier intended as a prototype for readout of silicon microstrip detectors at LHC which demonstrates the power of this approach. A CMOS circuit emulating the filter is being built. It has been implemented with extremely low power consumption $(<0.1 \mathrm{~mW} /$ channel $)$ in a small silicon area to be utilised on every channel of the system.
\end{abstract}

Submitted to Nuclear Instruments and Methods A

1) Institute of Nuclear Physics, Cracow, Poland

2) Blackett Laboratory, Imperial College, London SW7 2AZ, UK

3) Norwegian Institute of Technology, Trondheim, Norway

4) Senter for Industriforskning, Oslo, Norway

5) CERN, Geneva, Switzerland 


\section{INTRODUCTION}

The detection of events generated in proton-proton collisions at future hadron colliders will require the observation of interactions at intervals of $15 \mathrm{nsec}$. It is often assumed that the processing of signals from these events necessarily dictates the shaping of pulses in such a way that the pulse reaches its maximum amplitude within a single beam crossing interval (Fig.1a). Achieving this, even with a relatively small detector capacitance, has major implications for the choice of electronic technology, power consumption and electronic noise of the front end amplifier. It is therefore of considerable interest to evaluate alternatives to this approach.

For the readout of silicon microstrip detectors, one of the most promising position sensitive detectors for LHC type environments, the magnitude and time development of the available signal put stringent requirements on the front end design. Physics considerations limit the acceptable thickness of silicon detectors used for tracking to $\sim 300 \mu \mathrm{m}$, yielding a most probable value of $\sim 25000$ electrons for a minimum ionizing particle crossing the detector at 90 degrees. The effects of Landau fluctuations and charge sharing between strips, especially for steeply inclined tracks, imply that much smaller charges must be detected with high efficiency. Significant fluctuations from electronic noise in addition mean it is of paramount importance to maximise charge collection. However collection times, which in principle are only limited by carrier velocities in very high electric fields, are in practice determined by the fact that practical detector bias voltages will be in the range below 200 volts to achieve reliable operation of systems with many million channels. Detector current pulses with tails extending up to $20-30 \mathrm{nsec}$ may have to be tolerated.

With these constraints in mind, the possibility of using significantly longer amplifier time constants than the minimum value (Fig. 1b) is discussed. It is shown that it can offer some important benefits. A novel signal processing technique is described whereby the advantages of slow pulse shaping can be combined with those obtained using fast shaping, but using only a single amplifier time constant. Our analysis of this principle has shown that the technique may be applicable to a wider range of detector types than silicon microstrip detectors.

\section{LOW NOISE SIGNALPROCESSING ISSUES}

The basic principles of signal processing which determine the choice of amplifier time constant in a system where signal to noise is of paramount importance are described in several places[1-3]. In general analyses it can be shown that, in a system described by a 
time constant $\tau$, the equivalent noise charge $(\mathrm{ENC})$ is related to the detector capacitance in a simple way

$$
\mathrm{ENC}^{2}=\alpha \mathrm{C}^{2} / \tau+\beta \tau+\delta \mathrm{C}^{2}
$$

In this expression $\mathrm{C}$ represents the total capacitance appearing at the amplifier input and is generally dominated by the detector capacitance in most systems of interest for particle detection. $\alpha, \beta$ and $\delta$ are constants determined by the choice of technology and the noise sources characteristic of the amplifying elements employed. Modern electronic circuits are realised in either bipolar, MOS or JFET form; in practice JFET amplifiers are rarely used in integrated circuits and the choice is between bipolar and CMOS which are more readily available.

For low noise circuits in particle physics applications CMOS technology has become the preferred choice. This is partly for reasons of accessibility, but a factor of major importance has been the potential for achieving simultaneously adequately low noise and low power dissipation using CMOS. This is of great interest in high density applications, such as those employing silicon microstrip detectors. However, for applications where very fast response $(<15 \mathrm{nsec})$ is vital, bipolar transistor circuits can offer similar noise performance and improve on the power consumption attained by CMOS designs using short time constants[4].

The possible drawbacks of slow pulse shaping are higher shot noise after radiation damage to the detectors [5] and inferior timing resolution. In other respects it is desirable to work with CMOS technology for ease of access and cost, and thus reduced development time for new circuit designs. Also low power digital, and switched capacitor, circuits are easy to implement. Radiation hard fabrication processes exist, with potential application to low noise analogue circuits [6,7]. Finally, a CMOS design would be readily transferrable to a mixed bipolar-CMOS (BiCMOS) process if this technology becomes more accessible in radiation hard form in the next few years.

\section{THETIME AND AMPLITUDE PROBLEM}

Read out of detectors at high rates, with high efficiency for genuine events and excellent rejection of noise, requires the achievement of several goals simultaneously: sufficiently low noise for adequate signal discrimination, maximal charge collection and timing resolution which associates observed signals to a specific beam crossing. Whereas schemes with very fast shaping times and simple discriminator threshold cuts encounter problems with full charge collection and sufficient noise rejection, the approach of using 
slower shaping times and time over threshold discrimination [8] will make the timing requirements harder to realise due to inevitable timewalk.

An alternative approach is to recover the original impulse-like signal which generated the pulse shape we observe. Real signal impulses occur only at beam crossing times, while noise, which can give rise to both positive and negative going impulses, occurs at random moments in time.

We develop below the formalism necessary to recover an impulse signal from a shaped pulse and the implications for signal to noise. We demonstrate the results of applying the technique to measured signals from a low noise, low power CMOS amplifier which was developed as a candidate for silicon microstrip readout at LHC[9]. It is shown that better immunity to leakage current increases is provided by this method than by simply using a shorter time constant, while excellent timing resolution can simultaneously be obtained. In circumstances where leakage currents do not lead to excessive noise the advantage of full charge collection and lower noise signal measurement, obtained via slower shaping time, can be fully exploited.

The technique is applied by forming a weighted sum of the sampled output voltages of the amplifier from three successive time intervals. An important consequence of the method is that it can be implemented as an elementary CMOS circuit with negligible power consumption.

\subsection{The Deconvolution Principle}

The basic problem to be solved is: given an input signal, $s(t)$ and a knowledge of the impulse response of an amplifier, $h(t)$, how can the initial signal be retrieved from the measured output of the amplifier, $v(t)$ ? Fig.2 shows a schematic diagram of a system to do this.

It is well known that $\mathrm{v}(\mathrm{t}), \mathrm{s}(\mathrm{t})$ and $\mathrm{h}(\mathrm{t})$ are related in the time domain by a convolution integral. This can be written

$$
v(t)=\int_{-\infty}^{\infty} h\left(t-t^{\prime}\right) \cdot s\left(t^{\prime}\right) \cdot d t^{\prime}
$$

Here, we are particularly interested in the case when we sample the amplifier output voltage at regular intervals, so that the measurements of $v(t)$, for example, are $\left(v_{1} v_{2} v_{3} v_{4}\right.$ $v_{5} \ldots$...). Then it is convenient to write the equation in a matrix form ${ }^{1}$ as

1 Digital signal processing texts often express this equation in a linear form which, for our purposes, does not provide the same physical insight. 
or

$$
\begin{aligned}
& V_{i}=\sum_{j} H_{i j} S_{j} \\
& V=H . S
\end{aligned}
$$

The elements of $\mathrm{H}$ can be written down by inspection, taking into account the requirements of causality, which are that $h\left(t-t^{\prime}\right)=0$ for $t<t^{\prime}$, or $H_{i j}=0$ for $i<j$. Then, if $s(t)$ represents an initial impulse $\left(\begin{array}{lllll}1 & 0 & 0 & 0 & 0\end{array}\right)$ and the impulse response of the system is $\left(h_{1}\right.$ $\left.h_{2} h_{3} h_{4} \ldots\right)$ the resulting matrix equation is

$$
\left[\begin{array}{cccc}
h_{1} & 0 & 0 & 0 \ldots \\
h_{2} & h_{1} & 0 & 0 \ldots \\
h_{3} & h_{2} & h_{1} & 0 \ldots \\
\cdot & \cdot & \cdot & \cdot \\
\cdot & \cdot & \cdot & \cdot
\end{array}\right]\left[\begin{array}{l}
1 \\
0 \\
\cdot \\
\cdot \\
\cdot \\
h_{3}
\end{array}\right]
$$

Further inspection shows that this works for later impulses, like $\left(\begin{array}{lllll}0 & 1 & 0 & 0 & . .\end{array}\right)$ and $\left(\begin{array}{llll}0 & 0 & 1 & 0\end{array}\right.$ $0 .$. ) etc.

The original impulse can be reconstructed by performing the matrix inversion

$$
\mathbf{S}=\mathrm{W} . \mathbf{V}=\mathrm{H}^{-1} . \mathrm{H} . \mathbf{S}
$$

which is generally feasible, although in principle the matrix should be of infinite dimensions. ${ }^{2}$

Since $\mathbf{H}$ has a regular form (lower triangular matrix) it is clear that the inverted matrix, which we may call the weight matrix $\mathrm{W}$, is also very regular; it also has a lower triangular form. It must act on $\left(h_{1} h_{2} h_{3} \ldots\right)$ to give back the impulse response $\left(\begin{array}{lllllll}1 & 0 & 0 & 0 & 0\end{array}\right.$ ...) so that

2 In many cases $h_{1}=0$, eg the impulse response $-t e^{-t}$ of a charge sensitive preamplifier and CR-RC shaper, in which case the matrix $\mathrm{H}$ is singular. This can be avoided by the trick of writing

$$
\left[\begin{array}{cccc}
h_{2} & 0 & 0 & 0 \ldots \\
h_{3} & h_{2} & 0 & 0 \ldots \\
h_{4} & h_{3} & h_{2} & 0 \ldots \\
\cdot & \cdot & \cdot & \cdot \\
\cdot & \cdot & \cdot & \cdot
\end{array}\right]\left[\begin{array}{c}
1 \\
0 \\
0 \\
\cdot \\
\cdot \\
\cdot \\
h_{4}
\end{array}\right]
$$

and remembering that the impulse is displaced one unit of time from its true position. 


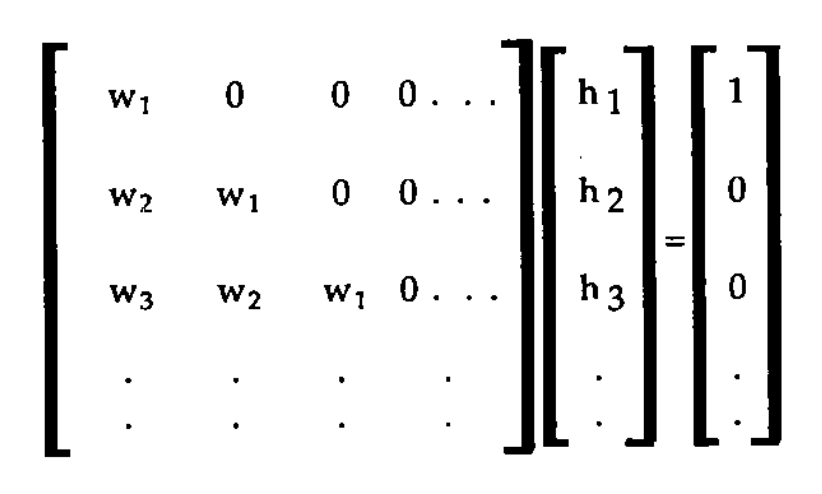

The weights $\left(w_{1} w_{2} w_{3} w_{4} \ldots\right)$ are required to recover the input signal from an arbitrary measured output signal $v(t)$. They can be calculated by solving the matrix equation analytically, in some simple cases, or be computed numerically if the impulse response is measured.

In general the weight vector $w=\left(w_{1} w_{2} w_{3} w_{4} \ldots\right)$ has an infinite number of elements. For a pulse shape of particular importance, produced by an ideal charge sensitive preamplifier followed by a CR-RC filter with equal time constants, it can be shown (see Appendix A) that only three non-zero weights are required. This offers the possibility of simple implementation of a circuit performing the deconvolution operation. Fig. 3 shows the result of performing this operation on an ideal pulse shape using the weights calculated in Appendix A.

\subsection{The Effect of Deconvolution on Noise}

There is a possible penalty associated with deconvolution because effectively the operation is equivalent to shaping the input signals using a filter which has a shorter time constant. Therefore parallel noise in the system is decreased while series noise increases.

It is shown below (Appendix B) that, for a CR-RC amplifier, the two components of the noise after deconvolution, $\sigma_{\mathrm{p}}(\mathrm{s})$ and $\sigma_{\mathrm{s}}(\mathrm{s})$, can be expressed as

$$
\sigma_{\mathrm{p}}^{2}(\mathrm{~s})=\left(\sigma_{\mathrm{p}} \mathrm{e}^{-2 / \mathrm{x}^{2}}\right)\left[\mathrm{e}^{2 \mathrm{x}}-4 \mathrm{x}-\mathrm{e}^{-2 \mathrm{x}}\right]
$$

and

$$
\sigma_{s}^{2}(s)=\left(\sigma_{s}^{2} e^{-2} / x^{2}\right)\left[e^{2 x}+4 x-e^{-2 x}\right]
$$

where $x=\Delta t / \tau$, is the ratio of sampling interval to amplifier time constant and $\sigma_{p}$ and $\sigma_{\mathrm{s}}$ represent the rms noise levels after the filter. The effect of different choices of $\mathbf{x}$ is shown in Fig.4.

If the pulse had simply been reshaped using a similar filter with a shorter time constant $\tau^{\prime}$ the consequences for the parallel and series noise are 


$$
\sigma_{\mathrm{p}}^{2}(\mathrm{~s})=\sigma_{\mathrm{p}}^{2} \mathrm{y}
$$

and

$$
\sigma_{\mathrm{s}}^{2}(\mathrm{~s})=\sigma_{\mathrm{s}}^{2} / \mathrm{y}
$$

where $y=\tau$ ' $/ \tau$. The results of this operation are also plotted in Fig.4. They show that deconvolution actually reduces parallel noise more than reshaping with a similar ratio of time constants $(x=y)$. Conversely, the series noise is increased slightly more than reshaping. For the choices of $\tau=45 \mathrm{nsec}, \Delta t=\tau^{\prime}=15 \mathrm{nsec}$ we find for the factor:

$\begin{array}{lcc} & \text { deconvolution } & \text { reshaping } \\ \text { Parallel noise } & 0.35 & 0.58 \\ \text { Series noise } & 1.84 & 1.73\end{array}$

Thus, provided the series noise of the system is sufficiently low, the deconvolution method offers greater immunity to increased shot noise from radiation induced leakage currents during LHC operation.

\section{DEMONSTRATION OF THE DECONVOLUTION METHOD}

\subsection{Signal and Noise Measurements}

To demonstrate the technique we have made use of a low noise, low power CMOS amplifier developed as a candidate for read out of silicon microstrip detectors at LHC[9]. The impulse response is very close to the ideal form produced by a charge sensitive preamplifier and CR-RC filter with equal time constants. Details of the performance of the amplifier are given elsewhere [9]; in its prototype form it achieves an equivalent noise charge of $390+67 \mathrm{C}_{\text {ext }}$ electrons, where $\mathrm{C}_{\text {ext }}$ is the external load capacitance in $\mathrm{pF}$, for a power consumption of $1.6 \mathrm{~mW}$ and a peaking time of $45 \mathrm{nsec}$.

Figure 5 shows a single waveform, in response to a fast test pulse, as measured on a digital oscilloscope with a sampling period of $15 \mathrm{nsec}$. The magnitude of the injected test charge was adjusted to be equivalent to 22,400 electrons; it originated from a pulse with a rise time $(10-90 \%)$ of less than $4.5 \mathrm{nsec}$. The oscilloscope samples were read via a standard interface (GPIB) and written to computer disk for subsequent analysis. Figure 6(a) shows the same waveform as Fig.5 displayed in the form of a histogram.

Deconvolution was carried out by means of a FORTRAN program using the weights calculated in Appendix B. For the data from the real amplifier plotted in Fig. 6(a) the result of the operation is shown in Fig. 6(b). The impulse signal is clearly recovered, with some increase in the noise as expected. The result is very similar to the deconvolution demonstrated for an ideal CR-RC shape shown in Fig. 3. 
More detailed studies were carried out to confirm that the behaviour of the noise after the deconvolution operation was in agreement with our predictions. To simulate the effect of leakage current a resistor was placed in parallel with the input of the amplifier in addition to a load capacitance of $7.5 \mathrm{pF}$; the equivalent leakage current was calculated from the resistor value using the relation

$$
\mathrm{I}(\mu \mathrm{A})=\frac{2 \mathrm{kT}}{\mathrm{qR}} \approx \frac{50}{\mathrm{R}(\mathrm{k} \Omega)}
$$

The results for three selected values of leakage current, $\mathrm{I} \approx 0 \mu \mathrm{A}, 4.1 \mu \mathrm{A}$ and $8.9 \mu \mathrm{A}$, are shown in Fig. 7(a)-(c). As shown in Appendix $B$, for $x=\Delta t / \tau=1 / 3$, we expect an increase in the series noise by a factor 1.85 and a decrease in the parallel noise by a factor 0.35 after deconvolution. Thus for $\mathrm{I} \approx 0$, when amplifier series noise dominates, we expect the noise to increase; we observe a factor 1.6. The discrepancy may be due to a contribution from $1 / \mathrm{f}$ noise in the amplifier, or from contributions to the total noise from elements after the first transistor, so that the simple model of parallel and series contributions is an oversimplification.

For large values of equivalent leakage current we observe decreases in shot noise by factors $0.43(4.1 \mu \mathrm{A}), 0.36(6.1 \mu \mathrm{A})$ and $0.35(8.9 \mu \mathrm{A})$ when the contribution of series noise is properly subtracted. These are in satisfactory agreement with our predictions. The signal to noise ratio is also in agreement with our estimates. Further measurements are under way to confirm the validity of the noise calculations under a wider range of conditions than examined so far; preliminary results are in good agreement with the calculations [14].

\subsection{Deviations from Ideal Behaviour}

So far we have demonstrated the deconvolution technique with reference only to ideal signal impulses. In practice, there will be deviations from this behaviour for a number of reasons.

Events occurring in subsequent beam crossings which generate signals in the same detector element will produce pile-up. For impulse signals this method is the most accurate technique for handling this problem since the intrinsic timing resolution is a single time slot. A deconvoluted signal is entirely confined to only one beam crossing interval and, provided the signal to noise ratio is adequate, the origin in time of two events occurring in subsequent beam crossings can be confidently identified. Figure 8 shows how this has been demonstrated with events separated by 30nsec. In the shaped pulse it is not possible to distinguish two individual events except by pulse height, which would be unreliable because of Landau fluctuations in a real detector. 
The quality of the timing information will depend crucially on the charge collection properties of the detector and will play a role in the performance of any fast electronic system. Simulations of charge collection in detectors have shown [10] that detectors should be operated with sufficiently high electric fields to ensure rapid charge collection. Such simulations give some guidance on the expected shape of current pulses and we have computed the performance of the deconvolution filter for a variety of input signals; one example is shown in Fig 9. This assumed an exponential current pulse ( $\mathrm{e}^{-t / T}$, with $\mathrm{T}=7.5 \mathrm{nsec}$ ). Some fraction of the input appears in subsequent time slots following the signal origin but not yet at a level which is intolerable. Further studies await the evaluation of the performance of this technique using detectors exposed to high levels of radiation where charge collection speeds may be degraded [11]. Of course, if the shape of the current pulse is well understood a choice of weights can be made which guarantees an impulse after deconvolution.

The quality of timing signals used for LHC or SSC operation will be of great importance to the performance of the final system. This is true whether or not the deconvolution technique is used. We believe this system will be robust to the variations expected in operation.

\section{IMPLEMENTATIONFOR HADRONCOLLIDER APPLICATIONS}

A schematic of the technique which indicates how it could be implemented in hardware is shown in Fig. 10. Sampled voltages from the shaping amplifier are multiplied by weights and summed to produce a signal to be compared against a chosen threshold. In practice, it is not desirable to filter all the data in this way since the $15 \mathrm{nsec}$ interval between bunch crossings implies an extremely high processing speed or a large degree of parallel processing to avoid interrupting the data flow. Moreover power consumption would be prohibitive in such a mode.

We propose to take advantage of the trigger hierarchy proposed for LHC to implement the technique as shown in Fig.11. A first level trigger is envisaged to be present after a $1-2 \mu \mathrm{sec}$ delay [12] with a positive Level 1 trigger decision made every $\sim 10 \mu \mathrm{sec}$. During the Level 1 trigger decision time sampled voltages from the amplifier are stored in an analogue delay buffer, or pipeline, typically 80 samples deep. In response to a positive Level 1 trigger three samples corresponding to the beam crossing for which the trigger occurred are transferred to the filter. This filter performs the analogue weighting and summing and gives a result within a delay of a few $\mu \mathrm{sec}$. This is sufficient to provide tracking information for use in a second level decision with a very small impact on the decision time. 
A circuit, based on switched capacitor techniques, running with a cycle time equal to the beam crossing interval, has been designed to perform this operation. A circuit schematic is shown in Fig. 12. It is referred to as an Analogue Pulse Shape Processor (APSP). If the APSP is idle when data arrive for processing it will start the filtering operation immediately. If it is still working on the previous event the data are stored in a queue. Thus, if the processing time of the filter is less than the average time between each Level 1 trigger, the system will be able to process all events (ie no deadtime) provided a sufficient number of buffers is available.

The design has been implemented in a $1.2 \mu \mathrm{m}$ CMOS process in an area of $800 \mu \mathrm{m} \mathrm{x}$ $40 \mu \mathrm{m}$ of silicon with a power consumption of $<0.1 \mathrm{~mW}$. It is thus suitable for inclusion on every channel of a very large silicon tracking detector.

\section{CONCLUSIONS}

The deconvolution method offers a very powerful method for signal processing at future high luminosity hadron colliders to provide both precise event timing and good quality analogue data, even in the presence of anticipated radiation damage. This method is very likely to be applicable in a wider variety of situations than we have yet explored. Deviations from ideal behaviour will occur when the initial signal differs significantly from a true impulse; in these circumstances the optimum strategy for processing the data is still to be defined.

\section{Acknowledgements}

We thank Rajiv Sachdeva for his assistance with experimentally verifying the noise calculations and Carole Ponting for her help in the preparation of this paper. 


\section{APPENDIX A-CALCULATION OF WEIGHTS}

The impulse responses of an ideal charge sensitive preamplifier plus standard CR$\mathrm{RC}^{\mathrm{n}}$ shapers, with equal time constants, are of the form

$$
h(t)=(t / \tau)^{n} \cdot e^{-t / \tau}
$$

For $n=1$ the weight vector $w$ becomes extremely simple since only three weights are nonzero. It is possible to see that this is true by noticing that the sampled values of $\mathbf{h}$ are of the form

$$
h_{n+1}=(n \Delta t / \tau) \cdot e^{-n \Delta t / \tau} \quad n=0,1,2,3, .
$$

$\Delta$ t being the sample period, so consecutive samples are

$$
(n-1) \cdot x \cdot e^{-(n-1) x} \quad n \cdot x \cdot e^{-n x} \quad(n+1) \cdot x \cdot e^{-(n+1) x}
$$

with $x=\Delta t \tau$, and it is necessary to satisfy equations like

$$
w_{k+1}(n-1) \cdot x \cdot e^{-(n-1) x}+w_{k} \cdot n \cdot x \cdot e^{-n x}+w_{k-1} \cdot(n+1) \cdot x \cdot e^{-(n+1) x}=0 .
$$

This can be achieved with weights which are related by

$$
\begin{array}{ll}
w_{1}=1 / h_{2} & =e^{x-1 / x} \\
w_{2}=-2 w_{1} \cdot e^{-x} & =-2 e^{-1 / x} \\
w_{3}=w_{1} \cdot e^{-2 x} & =e^{-x-1 / x} \\
w_{n}=0 & n>3
\end{array}
$$

after normalising $h(t)$ so that $h(\tau)=1$. For $\Delta t=15 n \sec$ and $\tau=45 n s e c, x=1 / 3$ and

$$
\begin{aligned}
& w_{1}=1.5403 \\
& w_{2}=-2.2073 \\
& w_{3}=0.7908
\end{aligned}
$$

Numerically these results can be obtained with great precision by matrix inversion, which offers a useful method for extraction of the weights for a non-ideal filter. For higher order shapers more weights are involved since equation (A3) has terms in higher powers of $\mathbf{n}$ than zero and one, so more coefficients are necessary. 


\section{APPENDIX B - NOISE AFTER DECONVOLUTION}

The evaluation of noise after the deconvolution operation is important for use of this type of filter. An unusual calculation is required since most treatments of noise and filtering refer to time continuous operations whereas here we are concerned with a system which is by definition based on sampling of a continuous waveform.

In general the deconvoluted signal at some arbitrary time, in the presence of noise alone, is given by

$$
s_{k}=\sum_{i} w_{i} v_{k-i+1}
$$

where $v_{i}$ are the noise output voltages. The mean (expectation) values of $v$ and $s$ are trivial since

$$
\left\langle\mathrm{s}_{\mathrm{k}}\right\rangle=\left\langle\mathrm{v}_{\mathrm{i}}\right\rangle=0
$$

We must determine the variance of $s_{k}$, which is

$$
\sigma^{2}\left(s_{k}\right)=<\sum_{i j} w_{i} v_{k-i+1} w_{j} v_{k-j+1}>
$$

Noise theory applies Campbell's theorem ${ }^{3}$ [13] to derive the rms noise after shaping as the sum of two components $-\sigma_{\mathrm{p}}$ and $\sigma_{\mathrm{s}}$ - representing parallel and series noise at the amplifier input $[1,2]$. It can be written

$$
\sigma_{\text {tot }}^{2}=\alpha \int_{-\infty}^{\infty}[h(t)]^{2} \cdot d t+\beta \int_{-\infty}^{\infty}\left[h^{\prime}(t)\right]^{2} \cdot d t=\sigma_{p}^{2}+\sigma_{s}^{2}
$$

where $h(t)$ is the response to a charge impulse. $\alpha$ and $\beta$ are coefficients which depend on the details of the noise sources[1,2]. Since they are uncorrelated we can treat the two contributions to the noise separately; for the CR-RC filter they can each be written as

$$
\begin{gathered}
\left\langle s_{k}{ }^{2}>=w_{1}{ }^{2}<v_{k}{ }^{2}>+w_{2}{ }^{2}<v_{k-1}{ }^{2}>+w_{3}{ }^{2}<v_{k-2}{ }^{2}>+2 w_{1} w_{2}<v_{k} v_{k-1}>\right. \\
+2 w_{2} w_{3}<v_{k-1} v_{k-2}>+2 w_{1} w_{3}<v_{k} v_{k-2}>
\end{gathered}
$$

or

$$
\begin{gathered}
\sigma^{2}(s)=\left(w_{1}{ }^{2}+w_{2}{ }^{2}+w_{3}{ }^{2}\right) \sigma^{2}+2 w_{1} w_{2} C(\Delta t)+2 w_{2} w_{3} C(\Delta t) \\
+2 w_{1} w_{3} C(2 \Delta t)
\end{gathered}
$$

3 Campbell's theorem states that in a linear system with impulse response $h(t)$ and a mean rate of random pulses $\lambda$, the average response is $\langle v\rangle=\lambda \int \mathrm{h}(\mathrm{t})$.dt with variance $\sigma^{2}(\mathrm{v})=\lambda \int \mathrm{h}^{2}(\mathrm{t})$.dt 
where $\Delta t$ is the sampling interval. The functions $C(\Delta T)$ give the average correlation between a measurement at some time and another delayed by $\Delta \mathrm{T} ; \mathrm{C}(0)=\sigma^{2}$. The autocorrelation function of $h(t)$, which determines the parallel noise, is 4

$$
C(\Delta T)=\alpha \int_{-\infty}^{\infty} h(t) \cdot h(t-\Delta T) \cdot d t
$$

For the CR-RC shaper this can be calculated

$$
C(y)=\sigma_{p}^{2}(1+y) \cdot e^{-y}
$$

with $y=\Delta T / \tau$. For large values of $y$, or samples well separated in time, $C(y) \rightarrow 0$, ie no correlation, while for $y \rightarrow 0 \mathbb{C}(y)->\sigma^{2}$, ie complete correlation - both as expected.

A similar calculation is required for the series noise using $h$ ' $(t)$ and this gives

$$
C(y)=\sigma_{s}^{2}(1-y) \cdot e^{-y}
$$

The two components of the noise after deconvolution can be expressed, after some algebra, as

$$
\sigma_{\mathrm{p}}^{2}(\mathrm{~s})=\left(\sigma_{\mathrm{p}} \mathrm{e}^{-2 / \mathrm{x}^{2}}\right)\left[\mathrm{e}^{2 \mathrm{x}}-4 \mathrm{x}-\mathrm{e}^{-2 \mathrm{x}}\right]
$$

and

$$
\sigma_{s}^{2}(s)=\left(\sigma_{s}^{2} e^{-2 / x^{2}}\right)\left[e^{2 x}+4 x-e^{-2 x}\right]
$$

with $x=\Delta t / \tau$, the ratio of sampling interval to amplifier time constant.

This analysis is strictly complete only in the absence of $1 / \mathrm{f}$ noise which is a good approximation for amplifiers of interest to us. A formal calculation of a $1 / \mathrm{f}$ contribution cannot be done in the time domain. However, from simple arguments, it can be surmised that the result will lie somewhere between the extremes of the series and parallel cases.

4 This can be proved in a similar way to Campbell's theorem. 


\section{REFERENCES}

[1] V. Radeka. Ann. Rev. Nucl. Part. Sci. $\underline{38(1988)} 217$.

[2] E. Gatti and P. Manfredi. La Rivista del Nuovo Cimento. $\underline{9}$ (1986) 1.

[3] P. Nicholson. Nuclear Electronics. J. Wiley (1974)

[4] H. Spieler. Power Requirements for Front-End Electronics in the Silicon Tracker. University of California, Santa Cruz, report SCIPP 91/28 (1991)

[5] G. Hall. Radiation resistance of semiconductor detectors and associated electronics. CERN 90-10 vol 3 (1990) 693.

[6] S. Tedja, H. H. Williams, J. Van der Spiegel, F. Newcomer, R. Van Berg. Noise Spectral Density of a Radiation Hardened CMOS Process in the Weak and Moderate Inversion. To be published IEEE Trans. Nucl. Sci.

[7] W. Dabrowski, M. Kajetanwicz, D. Kidwell, W. Rowe, H. Sadrozinski, E. Spencer, P. Tenenbaum, M. Turala, M. Wilder, H. Spieler. Noise Measurements on Radiation Hardened CMOS Transistors. University of California, Santa Cruz, report SCIPP 91/28 (1991).

[8] Conceptual Design Report for the SDC Silicon Tracker, (December 1991).

[9] T. Høgh, W. Lange, E. Nygård, P. Weilhammer, K. Yoshioka. A Low Noise, Low Power CMOS Charge Sensitive Amplifier for Silicon Detectors at LHC. To be presented at Milano Semiconductor Symposium (February 1992).

[10] S. Gadomski, M. Turala, A Zalewska, W. Dabrowski. CERN 90-10 vol 3 (1990) 241.

[11] H. Borner et al., The Development of High Resolution Silicon Strip Detectors for Experiments at High Luminosity at the LHC. CERN R\&D Proposal CERN/DRDC 91-10.

[12] N. Ellis and J. Garvey. CERN 90-10 vol 3 (1990) 80.

[13] N. R. Campbell \& V. J. Francis. Journ. Inst. Elec. Eng. 93 (1946) 45

[14] R. Sachdeva. Private communication. 


\section{Figure Captions}

Fig. 1 : Typical amplifier pulse shapes for (a) short shaping times ( $\tau=1$ ) and (b) longer shaping time pulse $(\tau=3)$. Time units are in beam crossing intervals (15nsec at LHC).

Fig. 2 : A schematic diagram of the filtering operations being applied in an amplifier system, including that necessary to retrieve an original impulse.

Fig. 3 : (a) The calculated output pulse from an ideal charge sensitive preamplifier and a CR-RC shaper with equal time constants $(\tau=3)$, sampled at every time interval, and (b) the result of a perfect deconvolution operation.

Fig. 4 : The effect of deconvolution on noise, expressed as the ratio of noise after processing compared to the original noise from the amplfier system. The solid lines show separately the consequences for parallel and series noise. The closed and open circles show the effect of simple reshaping with a CR$\mathrm{RC}$ filter with a shorter time constant $\left(\tau^{\prime} / \tau\right)$.

Fig . 5 : A single waveform from the amplifier descibed in the text, measured using a digital oscilloscope. The input was a fast risetime test pulse.

Fig. 6 : (a) The same waveform as measured in Fig. 5 shown as a histogram.

(b) The result of applying the deconvolution operation to the data from (a).

Fig. 7 : Noise distributions before and after deconvolution, measured as described in the text for different values of equivalent leakage current in the system. The leakage current was simulated by a parallel resistor at the input of the amplifier, where a $7.5 \mathrm{pF}$ load capacitance was also present. (a) $\mathrm{I}=0$, (b) $\mathrm{I}=4.1 \mu \mathrm{A}$, (c) $\mathrm{I}=8.9 \mu \mathrm{A}$. The shot noise was decreased by factors 0.43 and 0.35 , respectively after deconvolution, in the latter two cases.

Fig. 8. : Simulation of the amplifier response to two equal size pulses occurring separated by an interval of 2 time units. Here the data from individually measured sample pulses have been combined. (a) The result as measured at the amplifier output, (b) the result after deconvolution.

Fig. 9 : Simulation of the system response to a detector current pulse with an assumed $e^{-t / T}$ shape. $T=7.5 n s e c$ was used. (a) before deconvolution, (b) after deconvolution. 
Fig. 10 : A schematic of the deconvolution method. For the ideal pulse from a CR$\mathrm{RC}$ shaper only three weights are necessary to retrieve the impulse input. In more general circumstances more weights could be used. The lower part of the figure shows the consequence of applying the filter operation to events occurring one beam crossing interval before and after the one of interest, as well as to an event occurring in the beam crossing interval.

Fig. 11. : A schematic of the front end electronic system proposed for LHC. The amplifier/shaper is followed by an analogue pipeline which delays the samples for $1-2 \mu \mathrm{sec}$ until a Level 1 trigger has been received. If positive, the data are processed by the APSP.

Fig. 12 : A schematic circuit diagram of the APSP implemented in a switched capacitor form. 


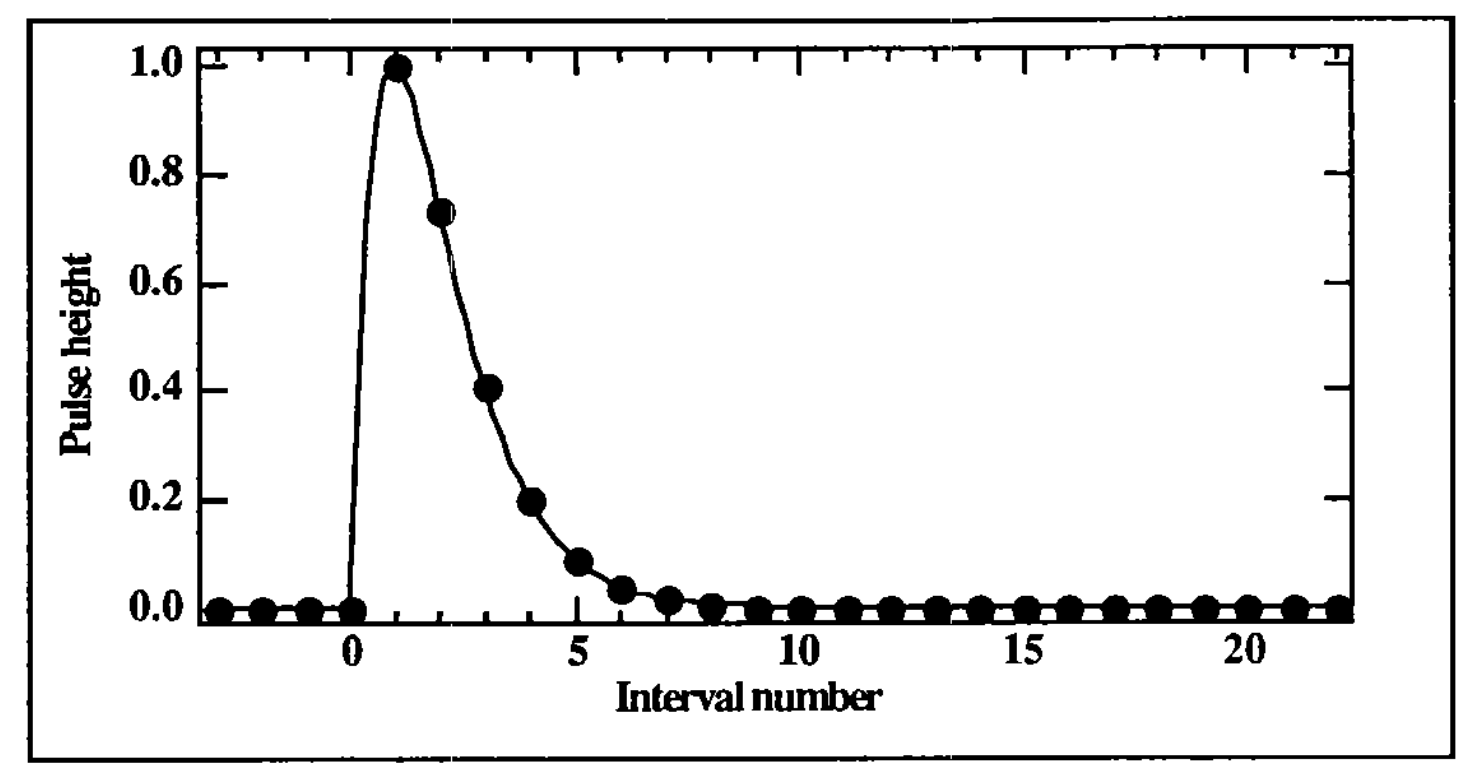

(a)

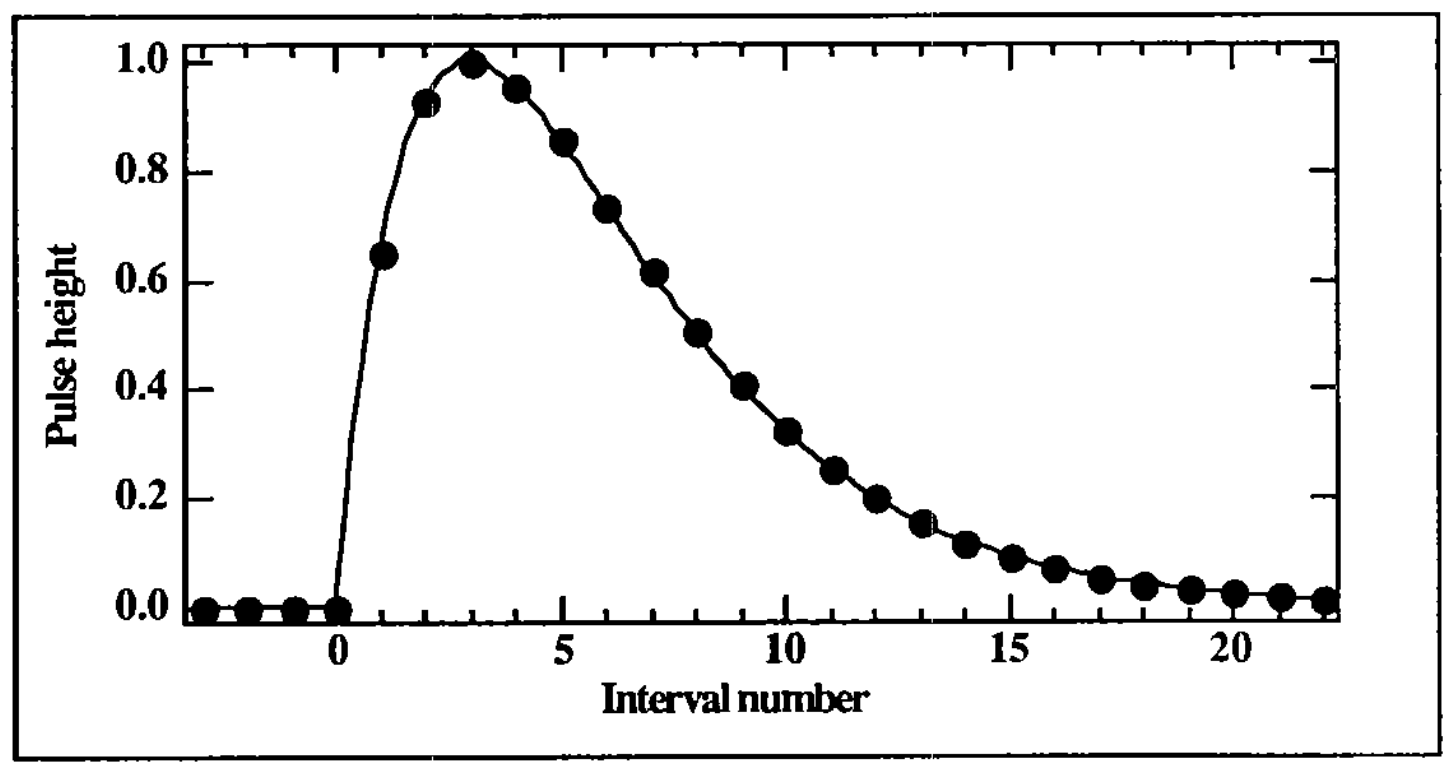

(b)

Fig. 1 


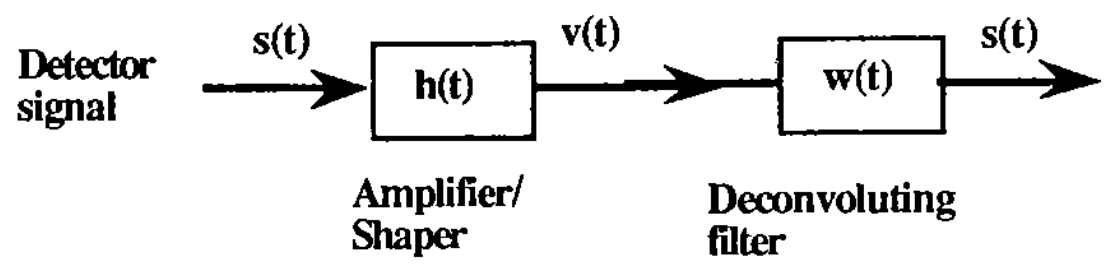

Fig. 2 


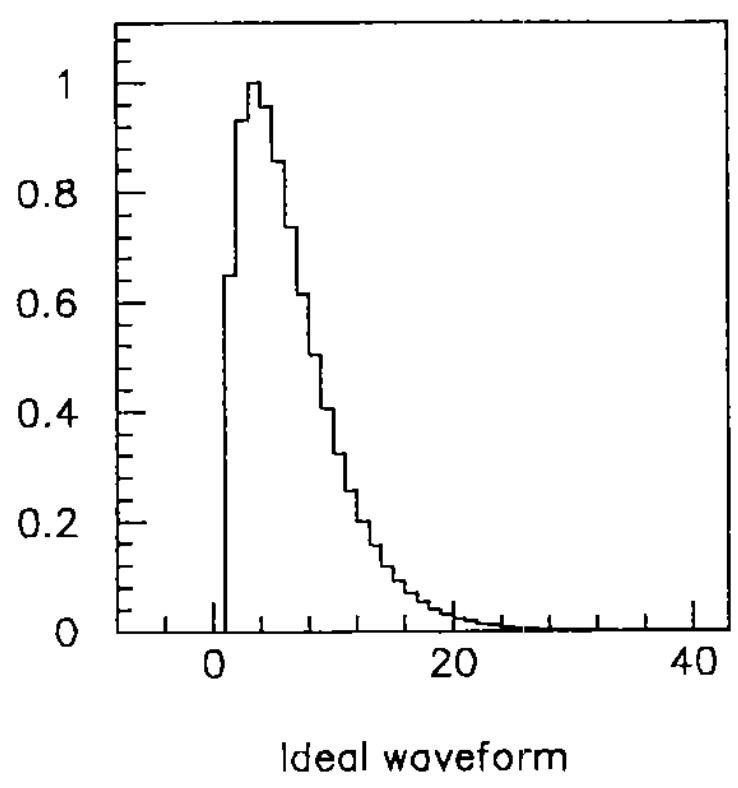

(a)

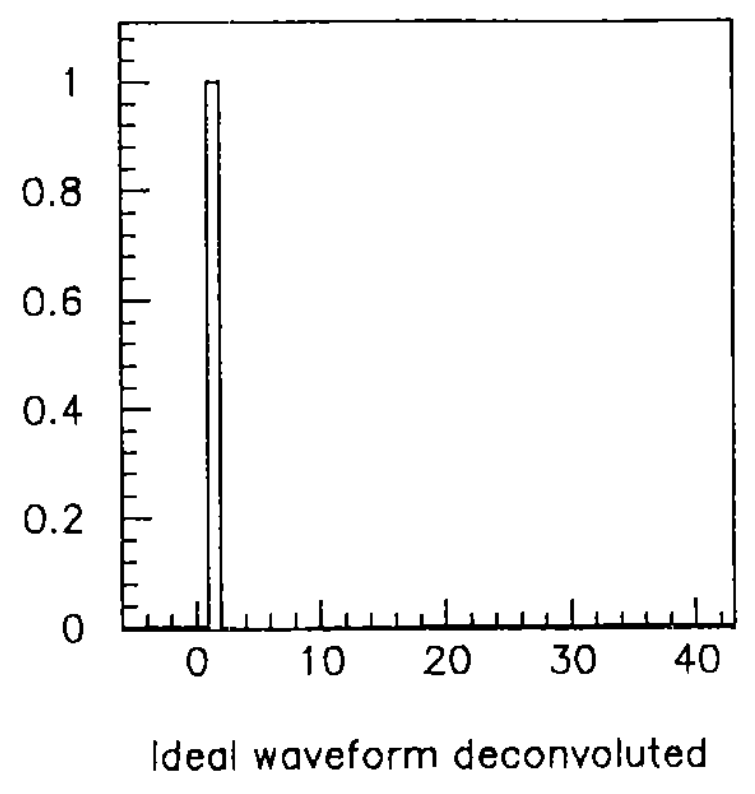

(b)

Fig. 3 


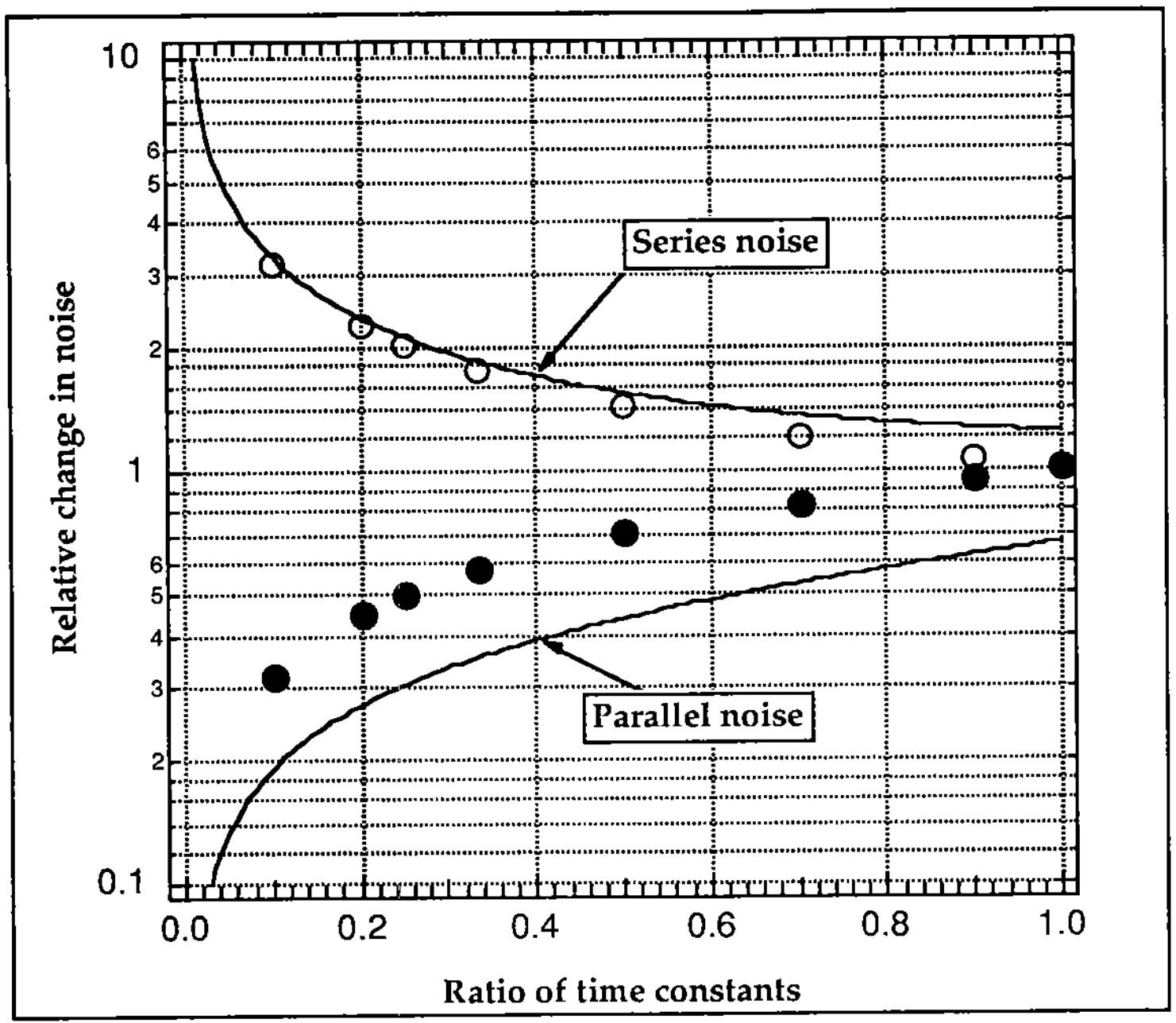

Fig. 4 


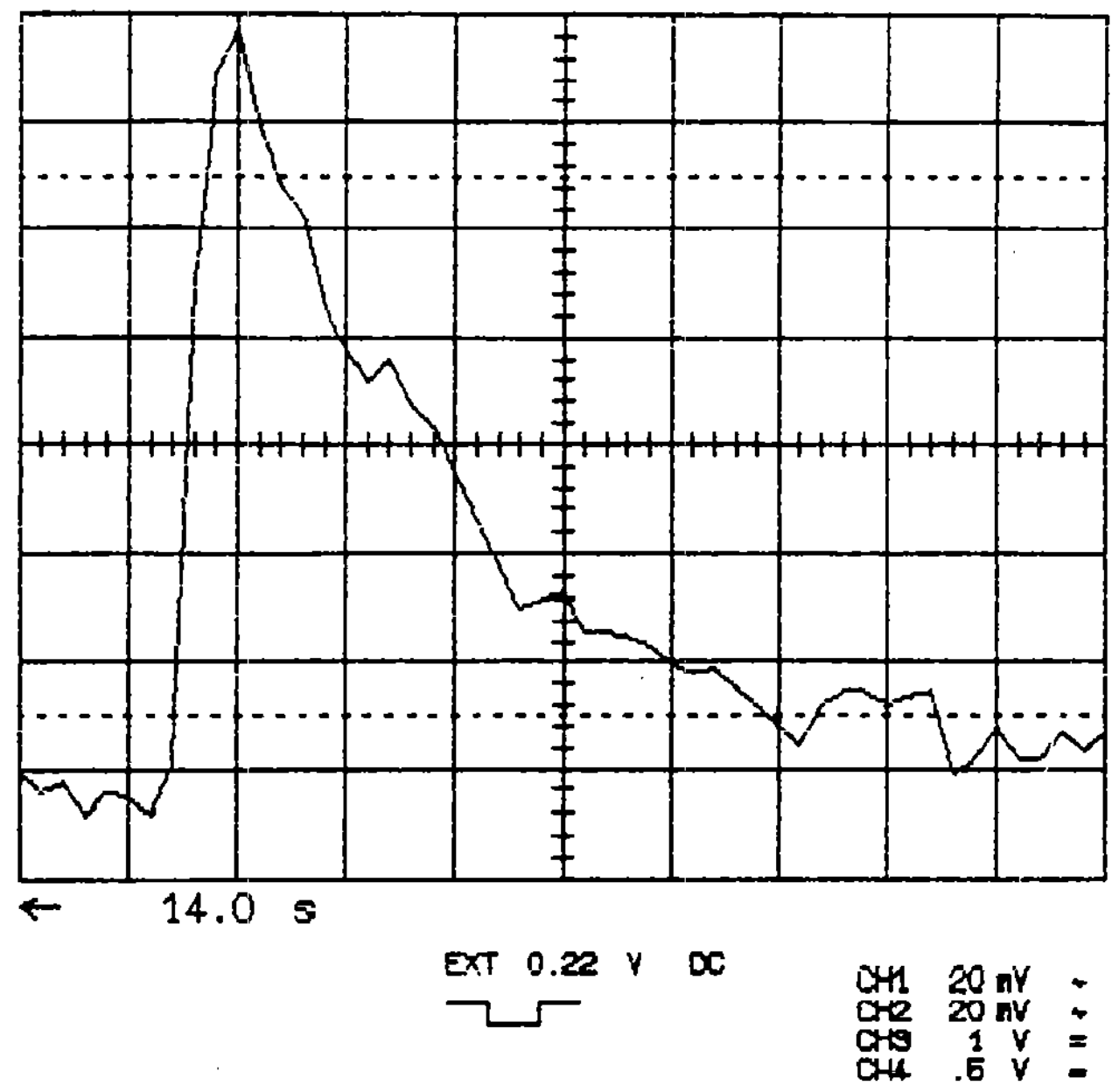

Fig. 5 


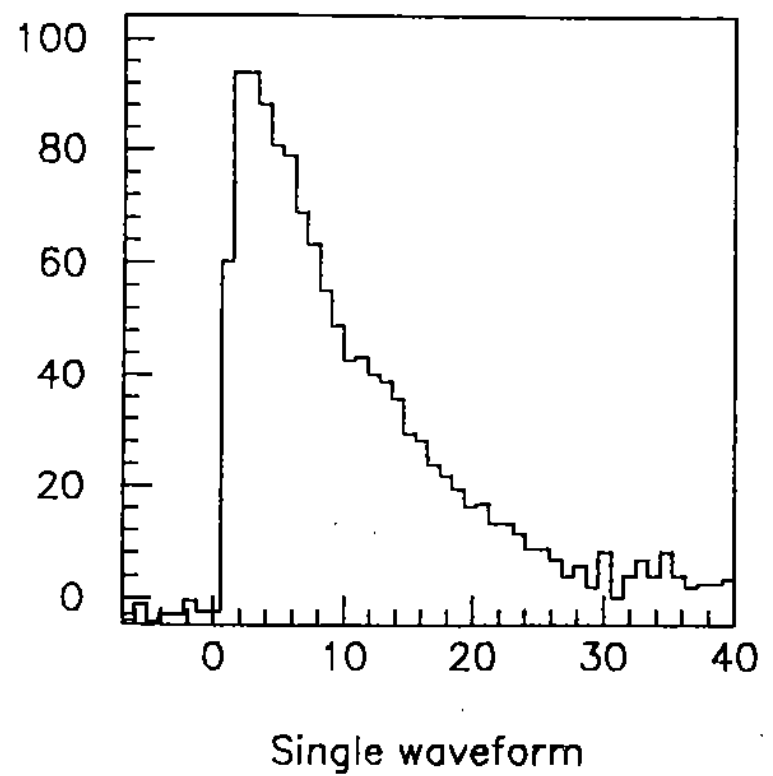

(a)

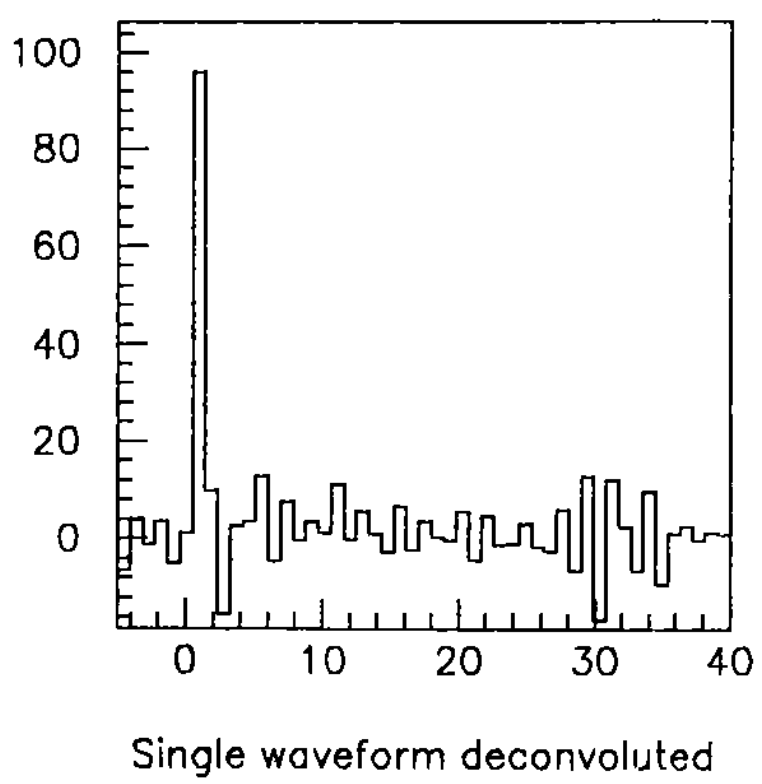

(b)

Fig. 6 
(a)

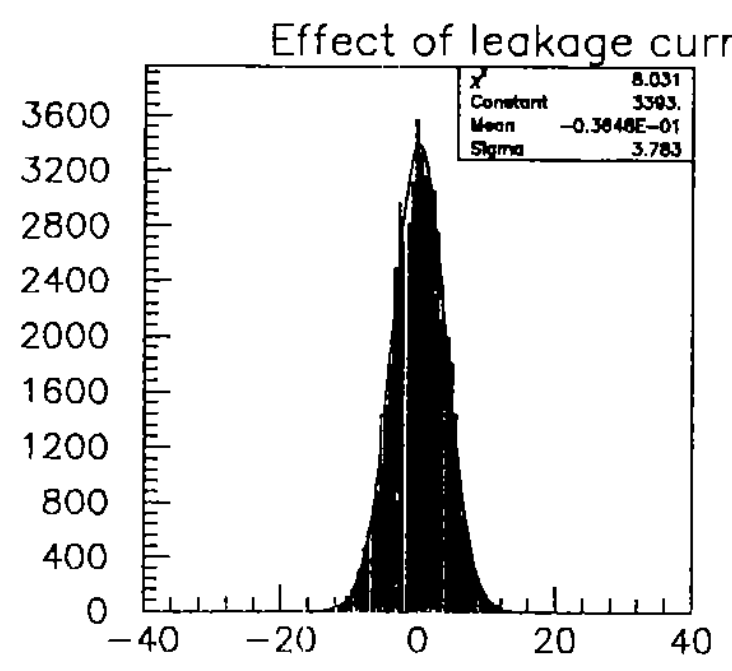

Noise distribution before deconv.

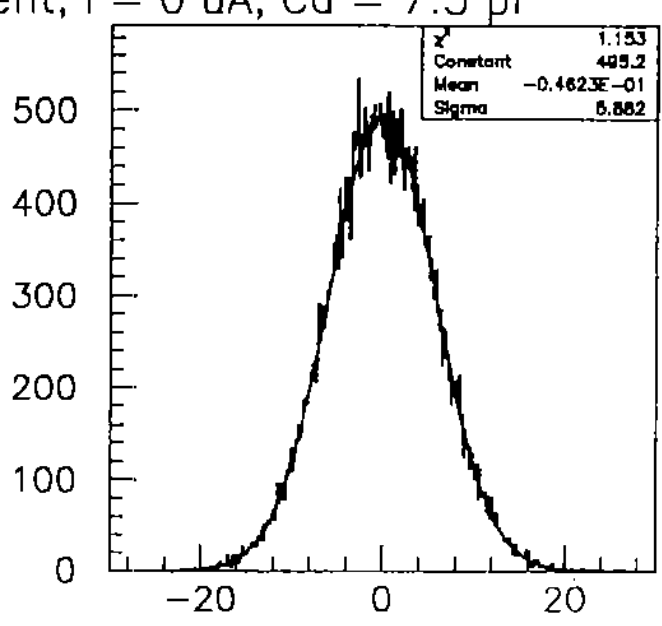

Noise distribution ofter deconv.

(b)

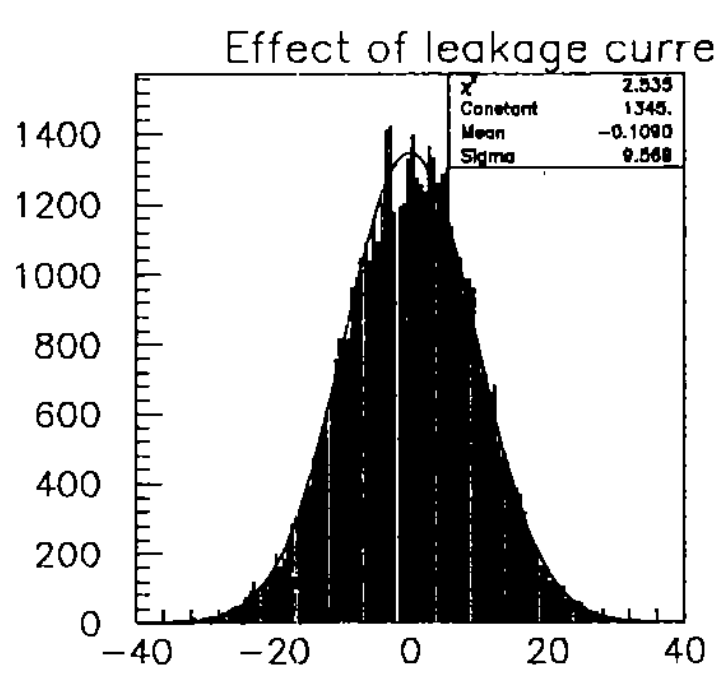

Noise distribution before deconv.

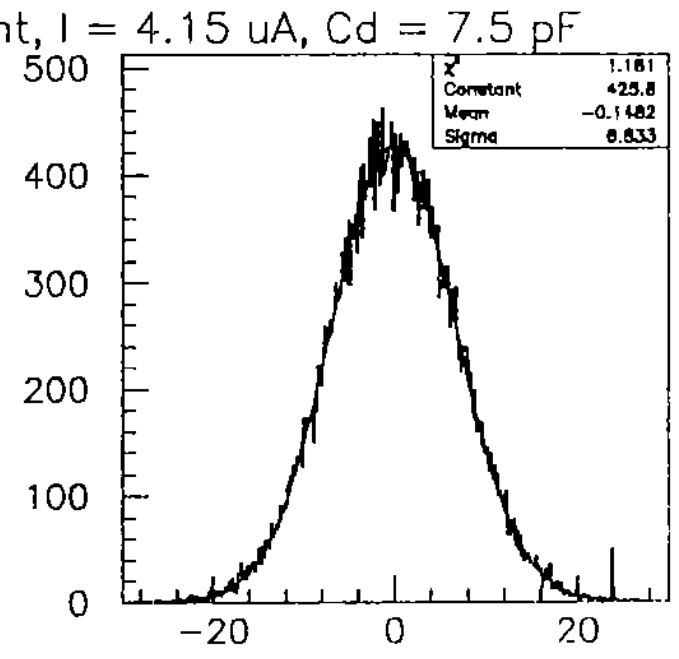

Noise distribution after deconv.

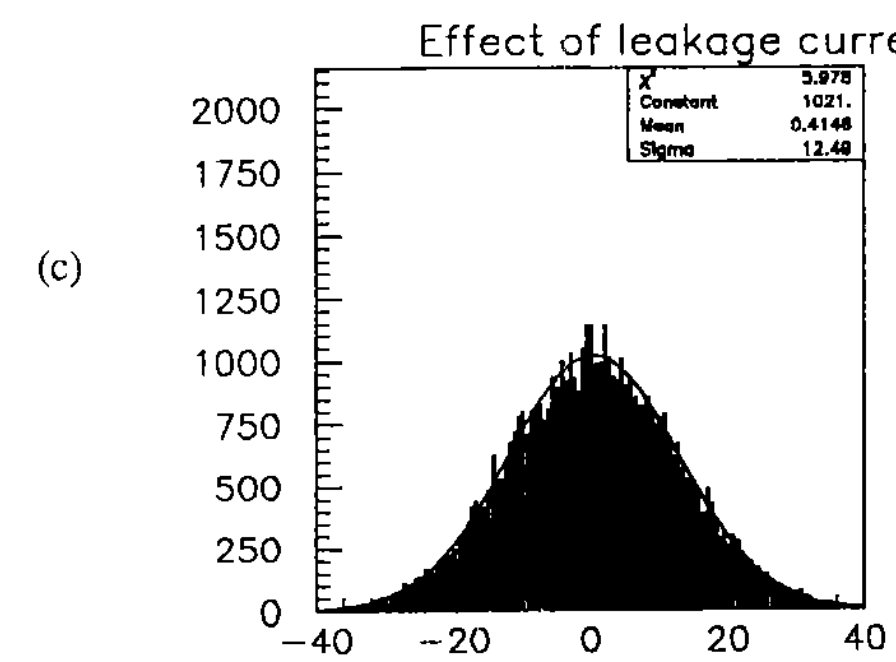

Noise distribution before deconv.

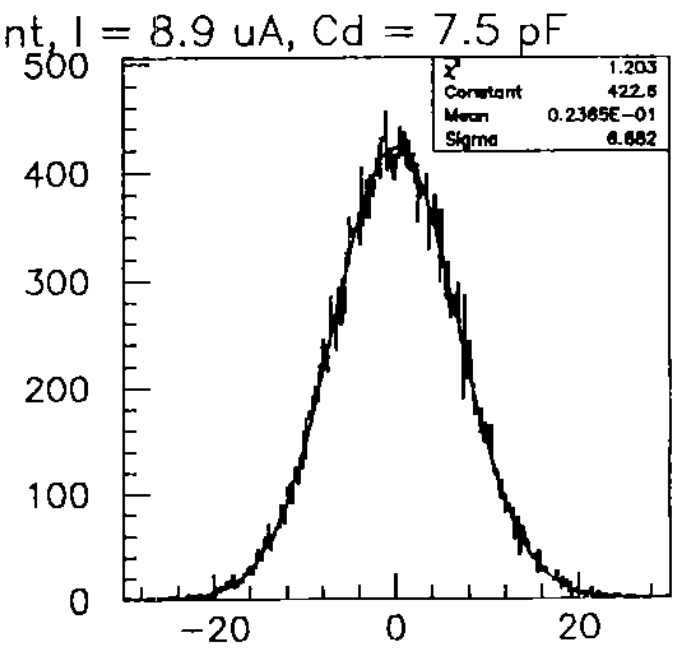

Noise distribution after deconv.

Fig. 7 


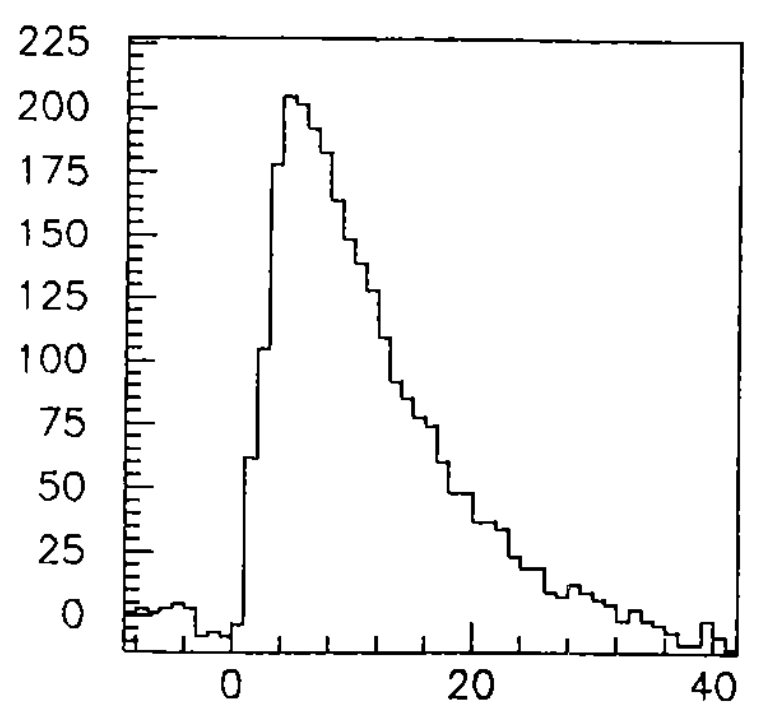

(a)

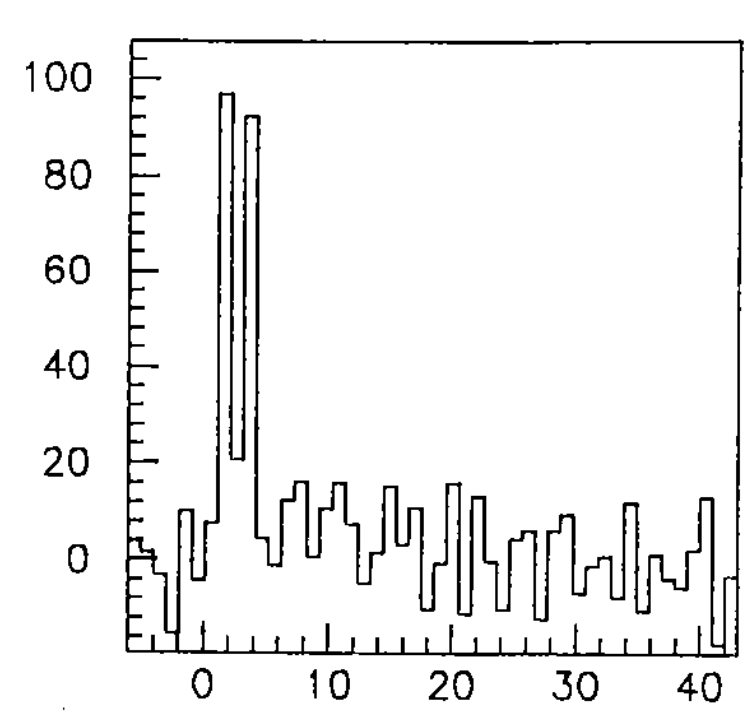

(b)

Fig. 8 


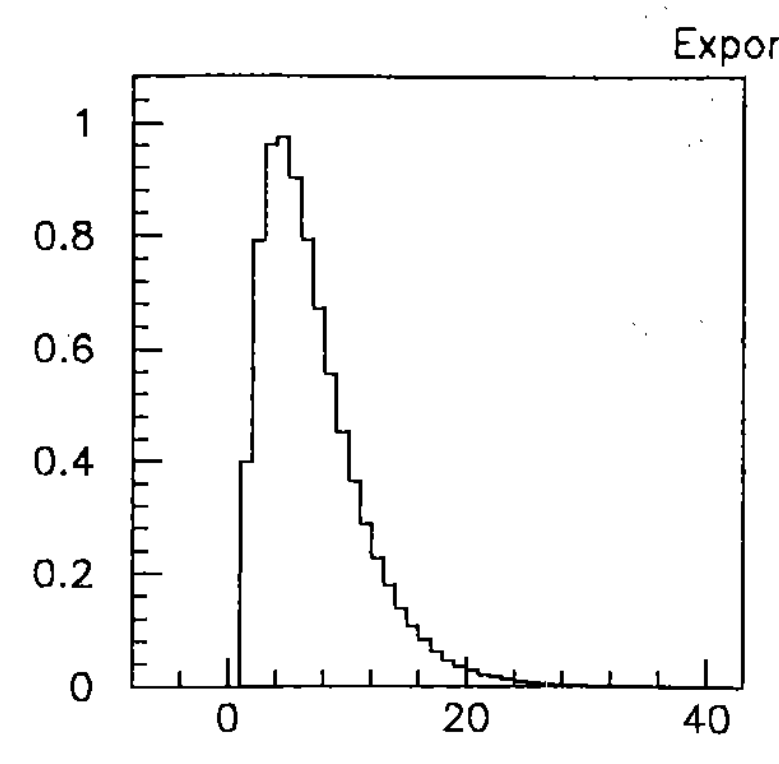

Before deconvolution, tau $=7.5 \mathrm{~ns}$

(a)

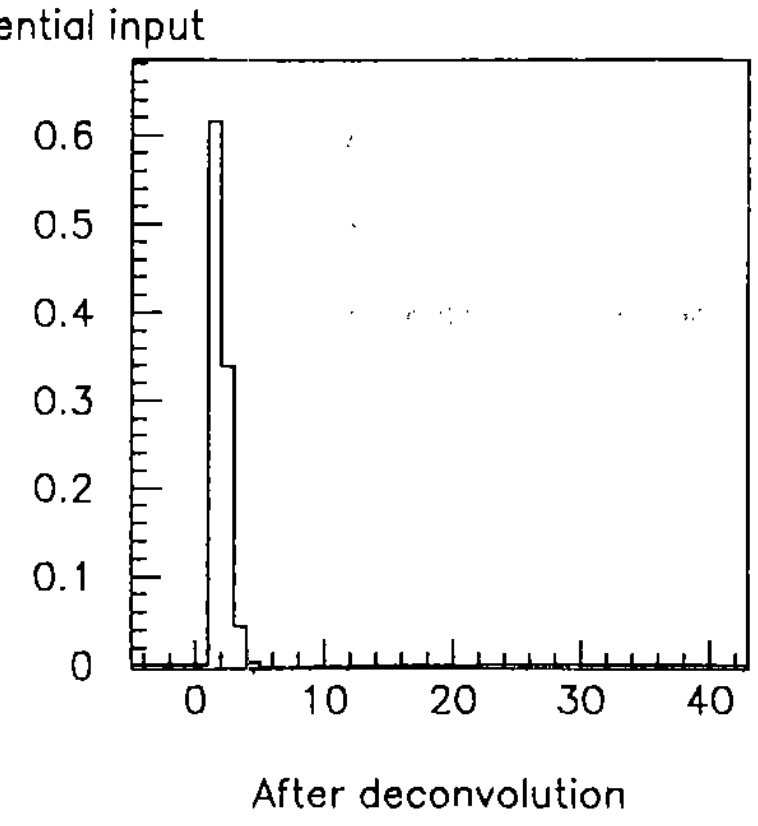

(b)

Fig. 9 


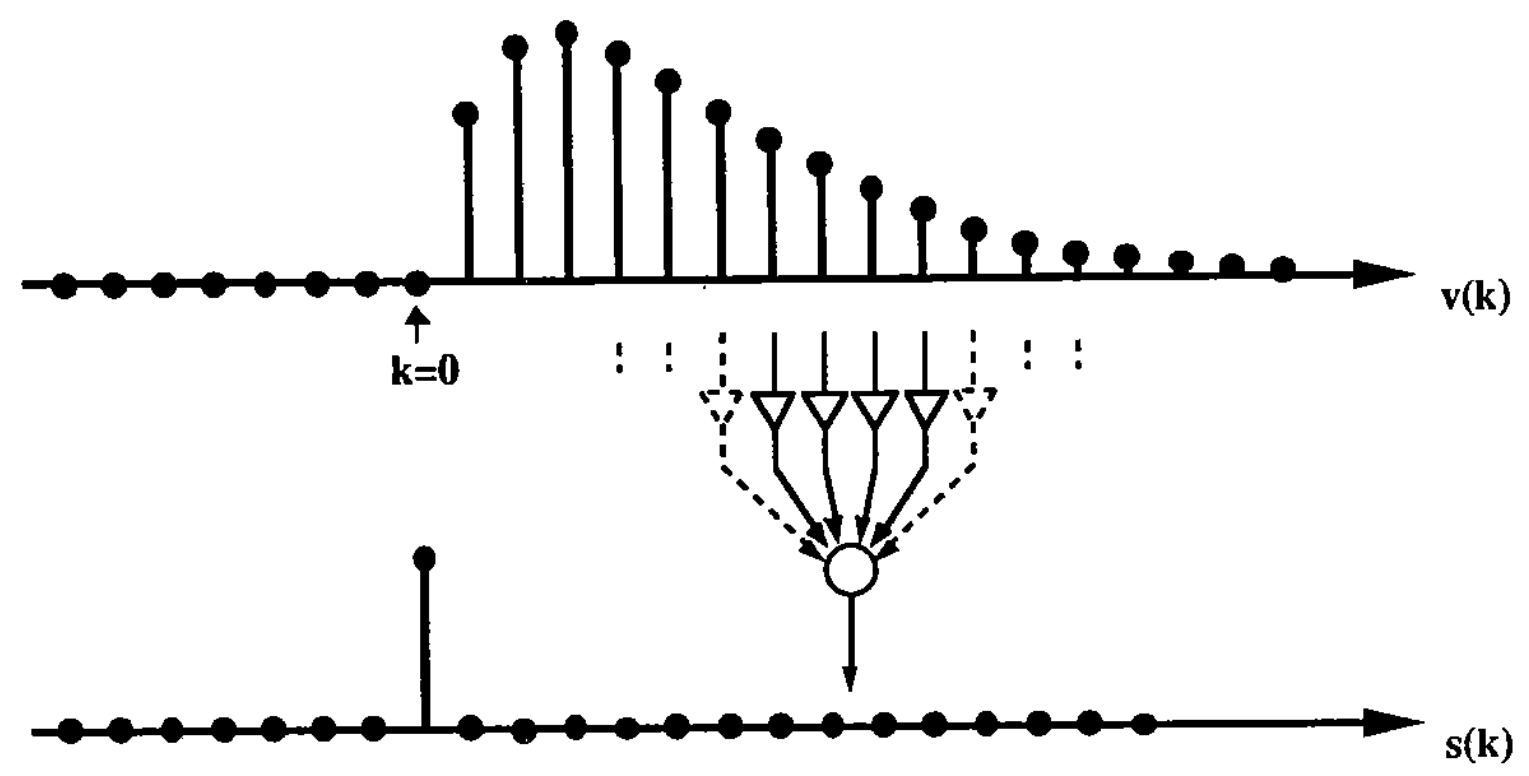

Signal at $t_{0}$
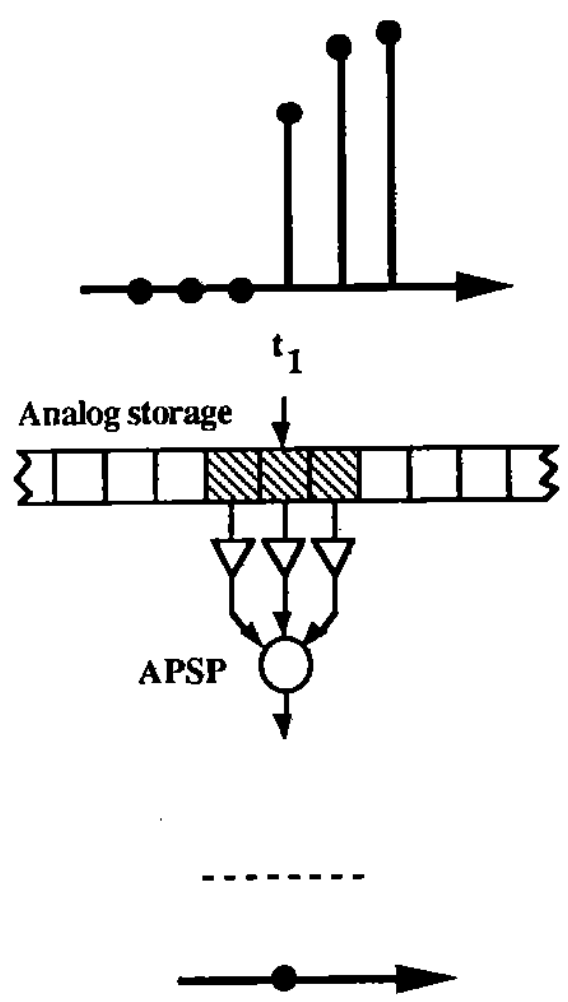

Signal at $t_{1}$
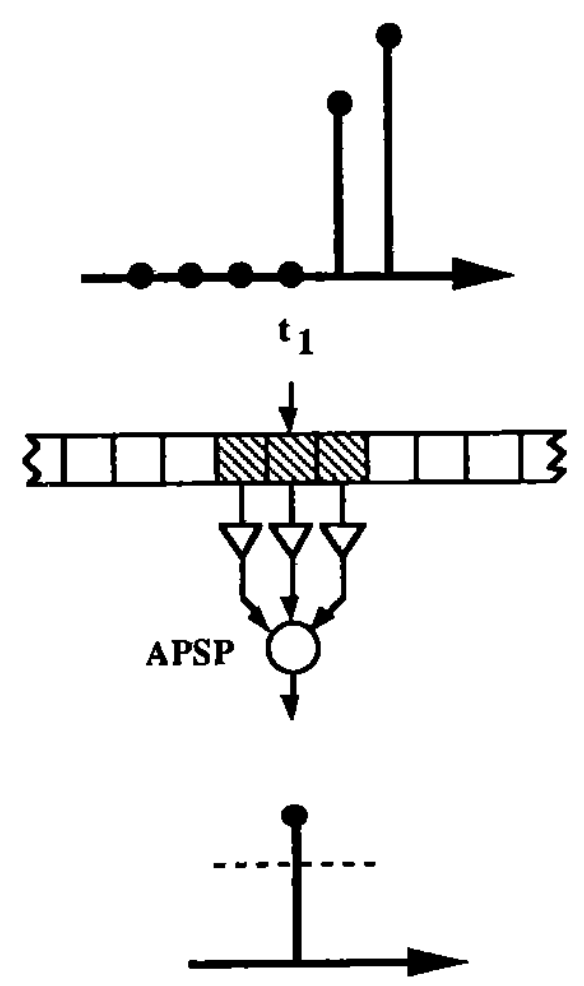

Fig. 10
Signal at $\mathbf{t}_{2}$
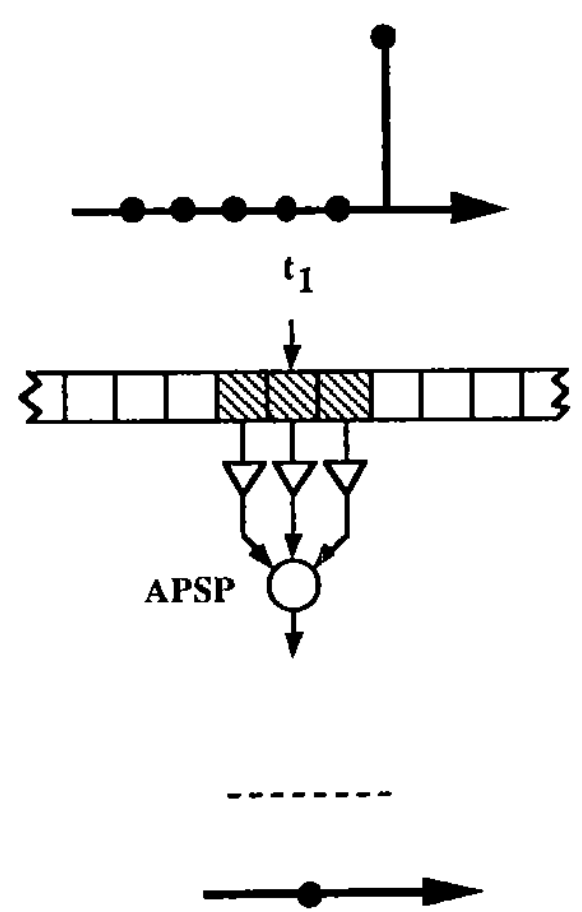


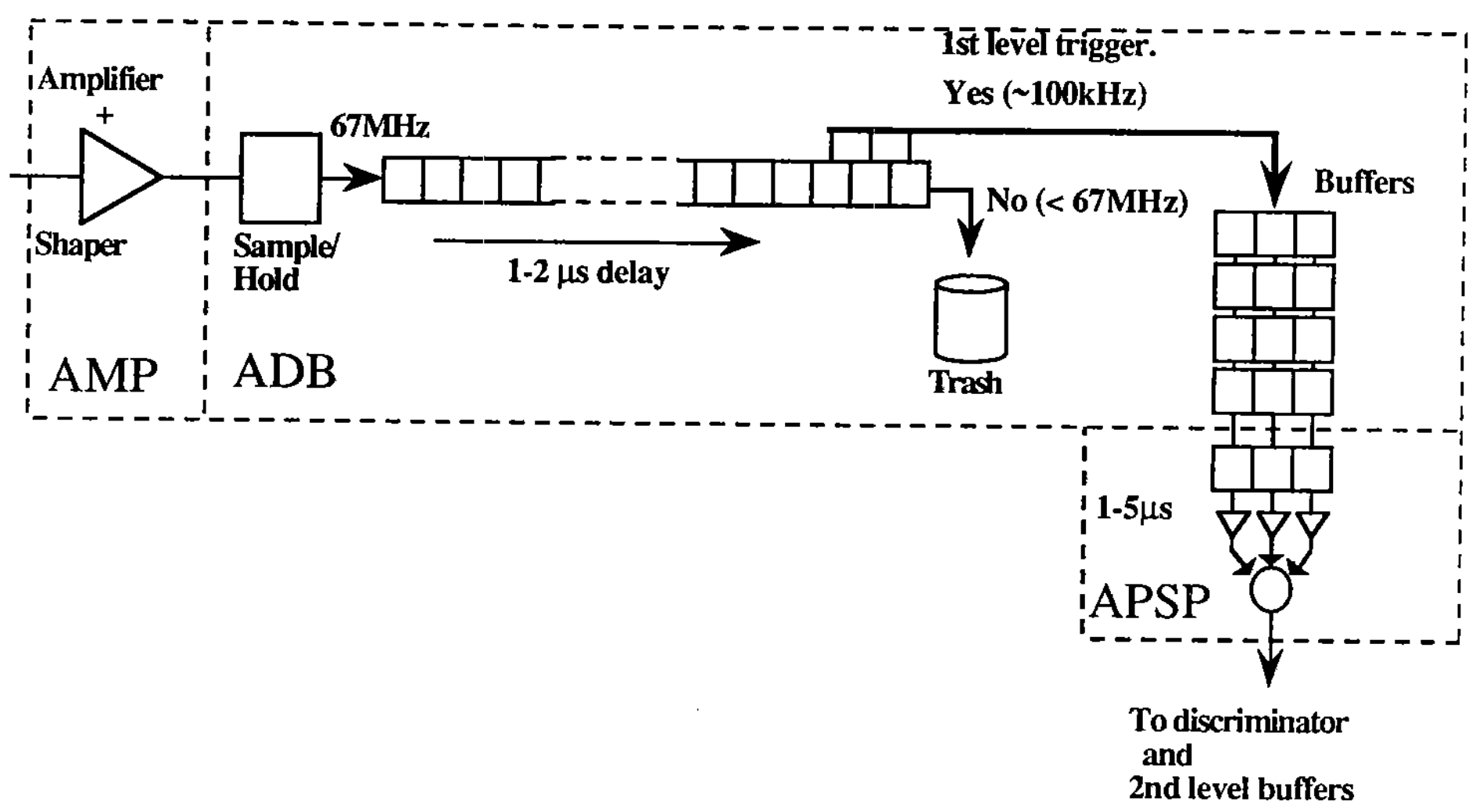

Fig. 11 


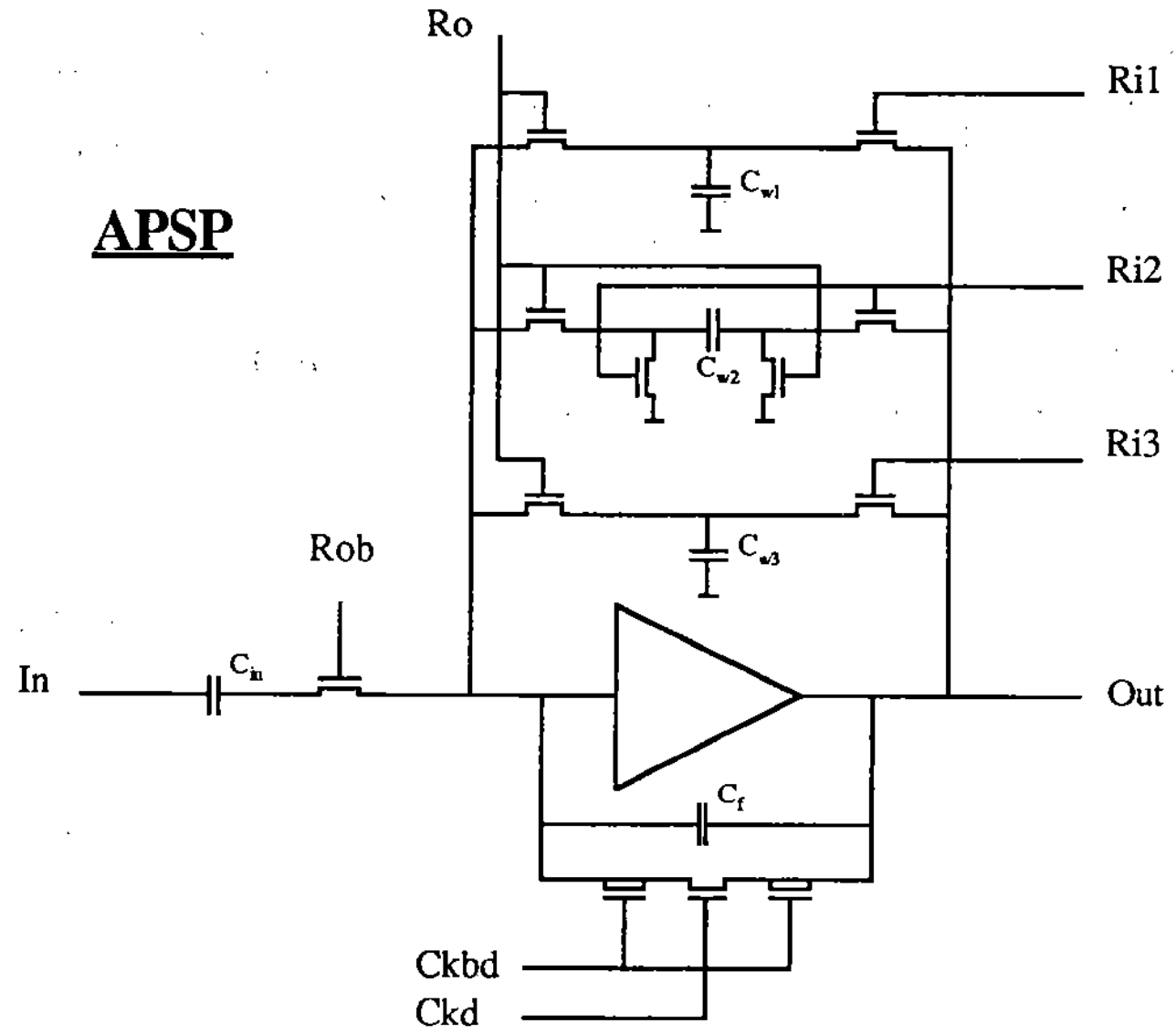

Fig. 12 